\title{
A Unified Heuristic X-ray Production Model for Thick and Thin Winds from Single Nonmagnetic Hot Stars
}

\author{
K. G. Gayley* \\ University of Iowa, 203 Van Allen Hall, Iowa City, IA 52242
}

\begin{abstract}
Observations of X-rays from WR6 and other dense winds require the presence of mechanisms that can produce hot gas at much larger radii than in lower density $\mathrm{OB}$ winds. But does this require some new mechanism in Wolf-Rayet winds, or could it simply be that the same hot-gas production is made more visible by denser winds? This article explores the latter perspective, and suggests a unified approach to the X-ray heating in all single nonmagnetic hot stars, as a kind of benchmark for observational testing. The results produce an X-ray generation efficiency that peaks as winds just become optically thick to X-ray reabsorption, but can still maintain detectable efficiencies at the large radii necessary in optically thick WR winds. A key element of the model is that fast terminal speeds serve to rapidly advect the gas being shocked out to large radii where some of the X-ray emission can emerge, even as X-rays emitted deeper down are copiously reabsorbed. An essential requirement is that the turbulence lengthscale increases with the wind acceleration lengthscale, as the latter is seen to be stretched out in Wolf-Rayet winds. Radiative efficiency is maintained at large radii by the high densities in the wind, allowing X-ray heating in an extended spatial "tail" of the normal OB-type emission to become observable. Hence in this scenario, the observation of dense winds serves as a complementary means of spatially resolving the nature of X-ray heating in nonmagnetic single hot-star winds.
\end{abstract}

Keywords: methods: analytical, X-rays, stars: mass loss, stars: winds, outflows, stars: Wolf-Rayet

\footnotetext{
${ }^{*}$ K. G. Gayley

Email address: kenneth-gayley@uiowa.edu (K. G. Gayley )
}

Preprint submitted to Advances in Space Research

January 10, 2016

(C) 2016. This manuscript version is made available under the Elsevier user license

http://www.elsevier.com/open-access/userlicense/1.0/ 


\section{Introduction}

Recent X-ray observations (Oskinova et al. 2012; Huenemoerder et al. 2015) of WR6 have demonstrated that the sources of X-rays in this wind are well outside the acceleration zone where the line-driven instability is expected to be active (Gayley \& Owocki 1995). A question that can be asked is whether or not this requires a fundamentally different process for generating these X-rays, than is active in less dense winds like those from $\mathrm{O}$ stars. Models of WR6 suggest that its wind is highly optically thick across the X-ray domain (Hamann et al. 2006), so we would not expect to be able to see X-rays from the acceleration zone. Thus we may find it surprising that WR6 is an X-ray source at all, and we may wonder if it possible for a similar mechanism that is responsible for X-rays in single nonmagnetic OB stars to also operate in WR winds, or if that is precluded by the observations. The similar mechanism would involve compressible turbulence stirred up in the acceleration zone by the line-driven instability (e.g., Dessart \& Owocki 2005a; Krticka et al. 2009) or some other instability inducing local variations in windspeed. The resulting dispersion in the windspeed would then need to survive to large enough radii to be responsible for strong shocks there.

Thus, the approach taken here is to examine general properties of X-ray generation in thick winds from the perspective that thick-wind X-ray generation is simply the extension of similar processes in thin winds, but where the thicker winds reveal a different aspect of those processes due to their higher X-ray emissivity at larger radii, coupled with copious X-ray reabsorption at smaller radii. The purpose is to explore the plausibility of unifying the mechanisms in nonmagnetic single stars throughout OB and Wolf-Rayet stars into a single idealized picture, and test that picture for any significant deviations from observations. As will be seen below, no clear contradictions with observations are seen using this picture if certain requirements are met, and several potentially elucidating features of thick winds, in contrast with thin ones, are encountered.

\section{Self-similar shock structure}

The approach here for unifying $\mathrm{OB}$ and WR wind X-ray generation is to assume that all these winds exhibit at radii well outside their acceleration 
zones a kind of two-phase structure, with clumpy slow wind moving at the prevailing terminal speed, and faster low-density streams filling the space between the clumps. This general type of structure, initiated by the linedriven instability, can be seen in one dimension in simulations like Feldmeier et al. (1997) and Runacres \& Owocki (2002), and in higher dimensions in Dessart \& Owocki (2005a). The way these winds will be unified here into a scale-free picture is by rescaling the radius by assuming the existence of a correlation length $L$ between the fast streams and slow clumps, embedded in the frame of the wind, such that the average time for fast wind to run into slow clumps is $t_{s} \cong L / \Delta v$, where $\Delta v$ is the characteristic speed excess in the fast streams. The $L$ distance scale parameter is the fundamental adjustable parameter in this model, and although heuristic at this point, it is intended to express a physical meaning, rather than represent a purely arbitrary parametrization of some kind of hot-gas filling factor.

Another advantage of the physical nature of the length $L$ is that it also corresponds to what has been called the porosity length in more formal clumping models (e.g., Sundqvist et al. 2012). It is expected here that this length may be roughly characterized by the size of the acceleration region in which the instability is active, on the grounds that the length scale on which bulk kinetic energy is pumped into the wind may also characterize the length scale on which turbulent energy is added by the instability. Thus we might expect $L \sim \beta R_{*}$, for $\beta$ an order-unity parameter that controls the size of the acceleration region.

For OB stars, $\beta \sim 1$ is common, but there is evidence that WR winds have larger $\beta$, such that $\beta R_{*}$ is in the range $10-20 R_{\text {/odot }}$ (Lepine \& Moffat 1999; Dessart \& Owocki 2005b). This implies large values of $\beta \lesssim 10$ if $R_{*} \sim 2--3 R_{\odot}$, as inferred for WN4 stars like EZ CMa (Hamann et al. 2006). The details of the appropriate value of $L$ will be left to future analysis, though it will be important for this model that it can be many stellar radii in WR winds, perhaps traced to their extended wind acceleration lengths. The approach here is to assume the $L$ parameter has been supplied, and then define a rescaled radius by $z=r / L$, such that $d z$ counts the fraction of a porosity length, which is the natural scale for tracking the shock physics. Even more importantly, the probability of the fast gas passing into a shock in scaled distance $d z$ is $d z / z_{s}$, where $z_{s}=v_{\infty} / \Delta v$ because the probability per time is $\Delta v / L$ by the definition of $L$, and the advected distance per time is $d r / v_{\infty}$ outside the acceleration region (which is the domain of the shock dissipation under consideration). Since it is assumed that the input shock 
structute initiates over the acceleration zone, the model used here assumes the wind is already clumped and already at $v_{\infty}$ throughout the domain where the shock dissipation is being tracked.

The assumption of a self-similar shock structure is then imposed by the expectation that at any scaled radius $z$, the mass fraction involved in fast streams is a universal function $y(z)$, which thus obeys the simple probabilistic relation

$$
\frac{d y}{d z}=-\frac{y(z)}{z_{s}}
$$

This admits the simple solution

$$
y(z) \propto e^{-z / z_{s}}
$$

in analogy to the well-known solution to the radiative transfer equation, where $z_{s}$ is the "mean free path" in $r / L$ units for shocking the fast streams. The range of the fast gas, for given $\Delta v$, is then described by $z \sim z_{s}$, which corresponds to the physical distance that the turbulent gas is advected in the time it takes to "catch up with" its clumpy slower targets over the distance $L$ in the clumpy wind frame. Higher $L$, or higher $v_{\infty}$, will tend to extend this advected distance, allowing hot gas to be created at potentially large enough radii to emerge through the photosphere of X-ray reabsorption.

\section{The Emergent X-rays}

The goal is to use the fast-gas fraction to infer the shock rate, and then the emergent X-ray flux, by noting that the emergent X-ray flux per unit radius, $d L_{x} / d r$, must obey the proportionality

$$
\frac{d L_{x}}{d r} \propto y(z) \frac{\Delta v}{v_{\infty} L} e^{-\bar{\tau}_{x}} F_{a d} F_{m i x}
$$

where

$$
e^{-\bar{\tau}_{x}}=\frac{1}{2} \int_{0}^{\pi} d \theta \sin \theta e^{-z_{1} \theta / z \sin \theta}
$$

accounts for X-ray reabsorption (MacFarlane et al. 1991) given that $z_{1}$ is the $z$ value where the radial optical depth is unity, and $F_{a d}$ and $F_{m i x}$ account for lost X-rays due to adiabatic cooling and mixing of hot and cool gas, respectively. We now turn to an estimation of these correction factors. 


\subsection{Reabsorption}

The expression for $e^{-\bar{\tau}_{x}}$, embedded in eq. (3), requires a double integration when the total X-ray emission is compiled over all radii. This is computationally inconvenient, given the level of approximation inherent in this heuristic approach. Let us instead define

$$
g(x)=-\ln \left(\frac{1}{2} \int_{0}^{\pi} d \theta \sin \theta e^{-x \theta / \sin \theta}\right)
$$

and note that eq. (4) implies that $\bar{\tau}_{x}=g\left(z_{1} / z\right) z_{1} / z$, where $g(x)$ varies slowly, so the dominant dependence of $\bar{\tau}_{x}$ on $z_{1} / z$ is drawn out explicitly. Then the integral in eq. (4) can be approximated to fairly good accuracy (generally within a few percent when it is important) by

$$
e^{-\bar{\tau}_{x}} \cong e^{-g(1) z_{1} / z}
$$

by taking as a characteristic value $g(1)=1.64$. The value of $z_{1}$ is proportional to the mass-loss rate $\dot{M}$, and also roughly to $\Delta v^{-4}$ if we assume the shock temperature scales with $\Delta v^{2}$ and the X-ray opacity scales roughly with the square of the photon energy (e.g., Leutenegger et al. 2010), under the assumption that the X-ray energy of interest is proportional to shock temperature. Thus we may define the parameter

$$
z_{\tau}=1.64 z_{1}=1.64 \frac{\dot{M}}{\dot{M}_{1}}\left(\frac{\Delta v}{700 k m s^{-1}}\right)^{-4},
$$

where $\dot{M}_{1}$ is defined to be the mass-loss rate such that the predominant Xray energies produced by a shock jump of $700 \mathrm{~km} \mathrm{~s}^{-1}$ (roughly $1 \mathrm{keV}$ in H-depleted WR winds) experience a radial optical depth of unity at $r=L$. The correction for reabsorption then becomes

$$
e^{-\bar{\tau}_{x}} \cong e^{-z_{\tau} / z}
$$

where $z_{\tau}$ is a kind of proxy for mass-loss rate in units similar to an X-ray optical depth of the terminal-speed wind, and depends sensitively on $\Delta v$, as seen in eq. (7). 


\subsection{Correction for adiabatic cooling}

Even before the X-rays are generated in the hot shocked gas, some of the energy can be shunted away by adiabatic cooling of the expanding gas. The expansion converts some of the thermalized energy back into bulk flow, but is assumed to do so in a way that largely dissipates the turbulent energy by mixing fast and slow streams. Thus it is regarded as a loss mechanism for the X-ray generating processes. The assumption here follows that of Owocki et al. (2013), which assumed that the adiabatic cooling rate is characterized by $v_{\infty} / r$. This means the primary expansion mechanism is assumed to be due to the spherical nature of the bulk flow, though this process if often of only minor importance except for very low mass-loss rates (characteristic of B stars). Owocki et al. (2013) found that the ratio of adiabatic cooling to radiative cooling is given by $z /\left(25 z_{1}\right)$, such that the correction for adiabatic losses in eq. (3) is

$$
F_{a d}=\left(1+\frac{g(1) z}{25 z_{\tau}}\right)^{-1}=\left(1+\frac{z}{15 z_{\tau}}\right)^{-1} .
$$

\subsection{Correction for mixing}

Also, if hot and cool gas mix, the ability to generate $\mathrm{X}$-rays is lost, and this loss channel is handled here by the mixing correction $F_{m i x}$. Again following Owocki et al. (2013), we have

$$
F_{\text {mix }}=\left(1+\frac{25 z_{\tau}}{g(1) z}\right)^{-m}=\left(1+\frac{15 z_{\tau}}{z}\right)^{-m}
$$

where $m$ is the "mixing exponent" that accounts for the tendency for mixing to occur when radiative cooling dominates and thin shells are created. The thin shells themselves must be already cooled by X-rays, but the dissipation of additional X-ray cooling occurs due to mixing of hot and cool gas across shear flows that set up around the thin shells (Kee et al. 2014). The latter authors find that for the highest mass-loss rates, a reduction in X-ray efficiency approaches a factor of 50, suggesting using a value for $m$ in the vicinity

of unity to achieve suitable X-ray reduction in the regions from which X-rays escape. 


\section{The X-ray emergence efficiency}

The above lays the groundwork to write the desired expression for the efficiency of X-ray generation and escape, written as

$$
\begin{aligned}
\eta_{X} & =\frac{L_{x}}{E_{x}} \cong \frac{\int_{1}^{\infty} d z e^{-z / z_{s}-z_{\tau} / z} F_{a d} F_{m i x}}{\int_{1}^{\infty} d z e^{-z / z_{s}}} \\
& =\frac{e^{1 / z_{s}}}{z_{s}} \int_{1}^{\infty} d z e^{-z / z_{s}-z_{\tau} / z}\left(1+\frac{z}{15 z_{\tau}}\right)^{-1}\left(1+\frac{15 z_{\tau}}{z}\right)^{-m}
\end{aligned}
$$

where $L_{x}$ is the emergent X-ray luminosity in the band of interest, and $E_{x}$ is the total excess energy flux injected into the wind in the form of turbulent velocity dispersion that is sufficiently hypersonic to produce X-ray hot shocks. One might imagine that $E_{x}$ represents a fairly fixed (small) fraction of the wind kinetic energy flux, though other scalings are also possible and are not the focus here. However, if $E_{x}$ is indeed proportional to mass-loss rate, then the way $L_{x}$ scales with mass-loss rate is modified by how $\eta_{X}$ scales with mass-loss rate. Also, $E_{x}$ does not include turbulent energy associated with weaker shocks that generate only EUV emission, and though future work can consider a spectrum of shock strengths, here the X-ray band is treated as a single separate component of turbulence.

The X-ray band of interest here involves shock strengths $\Delta v$ in the vicinity of $\Delta v=700 \mathrm{~km} \mathrm{~s}^{-1}$, characteristic of $1 \mathrm{keV}$ post-shock gas in low-H content WR gas. Since terminal speeds in the vicinity of $2000 \mathrm{~km} \mathrm{~s}^{-1}$ are also characteristic of WR winds with low $\mathrm{H}$ content, a putative set of parameters relevant here are $\Delta v=700 \mathrm{~km} \mathrm{~s}^{-1}$ and $v_{\infty} / \Delta v \cong 3$.

\section{Discussion}

Equation (11) is the fundamental result of this paper. Its form is clear; the efficiency $\eta_{X}$ is assumed to be controlled by the local processes that convert excess turbulent energy into emergent X-rays as per eq. (3), normalized by a denominator that leaves out the corrections for reabsorption, adiabatic cooling, and mixing, since the denominator represents all the energy dissipated in strong shocks stemming from the turbulent scale $\Delta v$, while the numerator limits to the energy that eventually emerges as X-rays. Hence, $\eta_{X}=1$ would imply that all the excess kinetic energy found in the fast gas in the comoving frame of the wind (i.e., the frame advecting at the terminal speed) 
is converted into emergent observable X-rays, whereas actual $\eta_{X}$ below unity quantifies the impact of reabsorption, adiabatic cooling, and mixing, all of which result in eventual energy escape in UV and EUV emission that are not involved in this analysis. The solution of eq. (11) is shown as a contour plot in Figure 1.

Figure 1 shows how the emergent X-ray efficiency, $L_{x} / E_{x}$, in some X-ray band of interest corresponding to shock strength $\Delta v$ (putatively $1 \mathrm{keV}$ X-rays for a shock strength of $700 \mathrm{~km} \mathrm{~s}^{-1}$ for H-depleted WR wind composition), depends on $z_{s}=v_{\infty} / \Delta v$ and $z_{\tau}=1.64 \tau(L)$. The first variable is proportional to terminal speed, once $\Delta v$ is fixed by the X-ray band of interest or the characteristic turbulence that is the source of X-rays, while the second is proportional to the mass-loss rate when all else is held fixed. The plotted X-ray efficiency does not include the initial efficiency in embedding turbulent energy into the clumpy bulk flow, so any total efficiency that considers the emergent X-ray flux as a fraction of the kinetic energy flux must combine both processes. This paper does not model the initial degree of energization, so that could be instead treated as a fixed constant fraction for all line-driven winds (perhaps on the order of $10^{-4}$ ), though if this is also treated as a variable, then some additional theory would be needed to constrain it.

The fraction of the turbulent energy that ultimately emerges as X-rays, $L_{x} / E_{x}$, is the efficiency of interest here, and Fig. 1 shows it is characteristically in the range 1-20\% for most of the winds in this model, when significant mixing $(m \gtrsim 2 / 3)$ is included. The primary lesson from Fig. 1 is that this efficiency shows a broad peak at intermediate mass-loss rates, which typically correspond to winds that are only marginally optically thin to reabsorption $\left(z_{\tau} \lesssim 1\right)$, perhaps of late $\mathrm{O}$ type. Earlier into the $\mathrm{O}$ type, and into the WR type at the top of the figure, the efficiency drops as the optical depth increases through unity, and the densest winds reabsorb a considerable fraction of their X-ray generation. By comparing $m=0$ and $m=1$ cases in Fig. 1 , we can also see that mixing can cause a similar drop in efficiency as does reabsorption.

Figure 2 recasts this efficiency, $L_{x} / E_{x}$, as a function of $\tau(L)$, the optical depth at $r=L(z=1)$, which is a proxy for mass-loss rate because of the direct proportionality seen in eq. (7). If we consider the putative value of interest $\Delta v=700 \mathrm{~km} \mathrm{~s}^{-1}$, appropriate for shock temperatures around $1 \mathrm{keV}$ in H-poor WR winds (Antokhin et al. 2004), then $\tau(L)=z_{1}=z_{\tau} / 1.64$. Thus Fig. 2 shows the effects of mixing, for increasing mixing exponent $m$, and motivates an expectation that $m$ in the vicinity of $2 / 3$ might yield the 


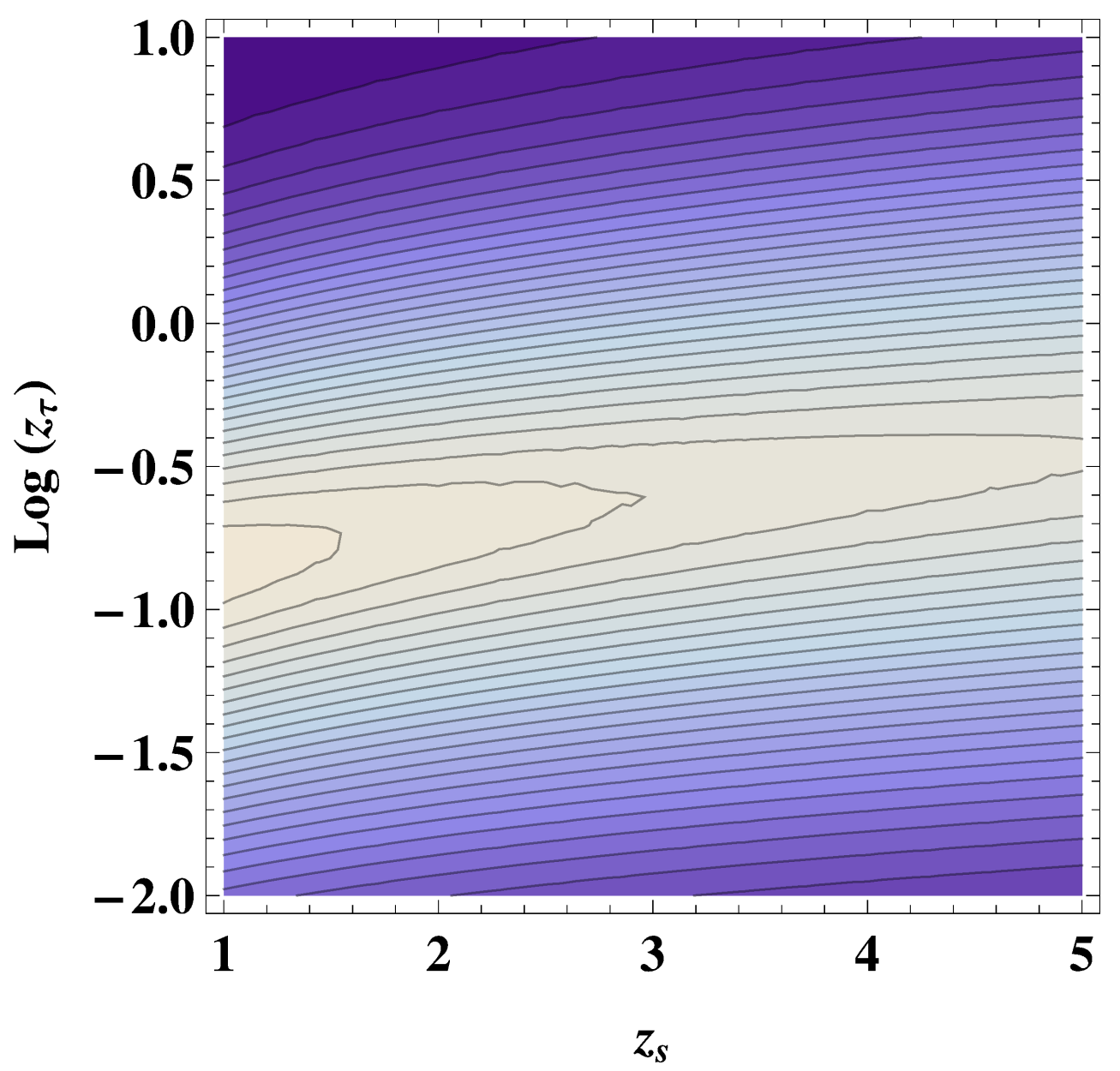

Figure 1: This figure shows contours of $L_{x} / E_{x}$ (solid curve), as a function of $z_{s}$ and $\log _{10} z_{\tau}$, for mixing exponent $m=2 / 3$. The maximum contour in the center is $29 \%$, and each contour steps down by $1 \%$, reaching very low efficiency in the upper left corner. The low X-ray efficiency at low $z_{\tau}$ is due to adiabatic cooling at B-star mass-loss rates, and the low efficiency at high $z_{\tau}$ is due to X-ray reabsorption at WR mass-loss rates. The lower efficency at low $v_{\infty}$ for high mass-loss rates (high $z_{\tau}$ ) is due to the inability of slow winds to advect the turbulent gas to the region beyond the X-ray reabsorption photosphere. The higher efficiency at low $v_{\infty}$ for low mass-loss rates (low $z_{\tau}$ ) is due to reduced adiabatic cooling in the higher densities of low-speed winds. Lower mixing exponents $m$ increase the efficiency, and higher $m$ reduces it even more. 
best results for this model. This evidence comes from observations of Xray luminosity that were found in Owocki et al. (2013) to best fit the thin line in Fig. 2, where this line is extended into the WR regime following the observational results of Skinner et al. (2012). Those observational results suggest that $L_{x}$ is fairly independent of $\dot{M}$ for $\mathrm{H}$-poor WR winds, requiring that $L_{x} / E_{x} \propto \dot{M}^{-1}$ if the initial energization fraction is assumed to be fairly constant.

These results are only intended to guide further investigation; appropriate values for $m$, or a different mixing parametrization altogether, will require a more careful analysis of simulations and observations. Initial work in that direction by Owocki et al. (2013) suggested $m \sim 0.4$, though followup work by Kee et al. (2014) found that a larger $m$, with possible saturation at a mixing correction of $1 / 50$, may be more appropriate. At this point, both the reduction in X-ray efficiency due to mixing, and the total efficiency for converting turbulent energy into X-rays, both require further study, and the modular approach to mixing here is flexible enough to be replaced as necessary.

Also of interest is how the efficiency $L_{x} / E_{x}$ depends on mass-loss rate at various different shock strengths for fixed terminal speed. Figure 3 varies $\Delta v$ in both the $z_{s}$ and $z_{\tau}$ parameters (the latter because the optical depth is assumed to drop steeply like $\Delta v^{-4}$ since harder emissions from stronger shocks emerge more easily), to show that stronger shocks produce better overall X-ray efficiency. This holds even though the faster gas needed for stronger shocks overtakes the terminal-speed clumps more rapidly, so has a shorter advection range, and the X-rays must thus emerge from deeper in the wind. That the opacity is so much less at higher energy is why that competition is successful for the stronger shocks, suggesting that high-density winds should be harder sources than might otherwise be expected in analogy to $\mathrm{OB}$ winds. In this regard, it is interesting that observations of WR6 do find evidence (Oskinova et al. 2012) for relatively harder emission, though certainly other models could also produce such an effect.

One of the most important findings of the WR6 X-ray observations (Oskinova et al. 2012; Huenemoerder et al. 2015) is that the X-ray emission comes from far out in the wind, perhaps 100 stellar radii depending on X-ray energy. This is inferred from a need for low EUV irradiation in models of He-like ions with "f/i/r" multiplets, and is consistent with the expectation of high optical depth to reabsorption in deeper wind layers. Figure 4 shows the spatial distribution (in rescaled $z$ space) of the contribution to the X-ray 


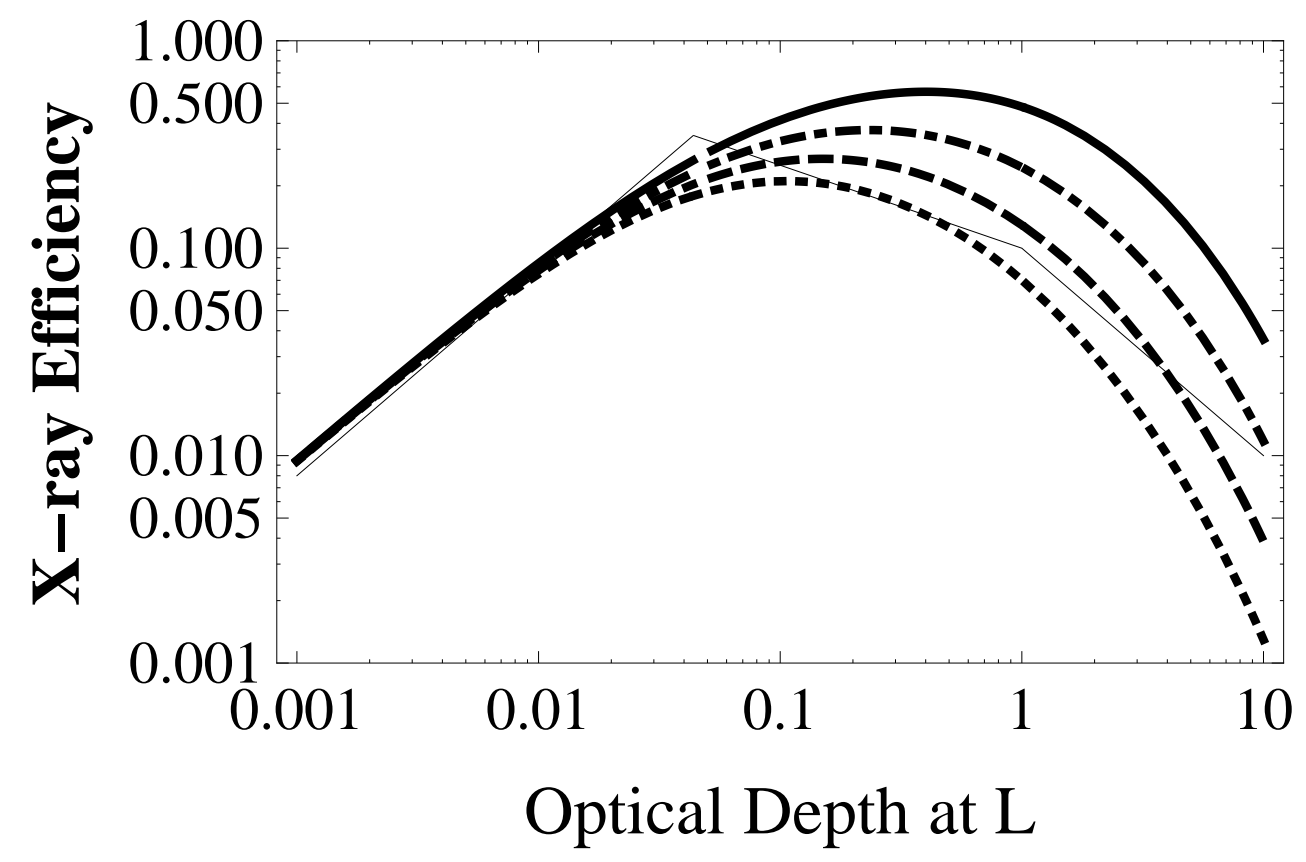

Figure 2: Here the X-ray efficiency $L_{x} / E_{x}$ is plotted as a function of mass-loss rate characterized by $\tau(L)$, the optical depth to X-ray reabsorption at $L$ in the X-ray band of interest $(\sim 1 \mathrm{keV})$, using various choices for the mixing exponent $m$. The solid curve has $m=0$ (no mixing), the dot-dashed curve has $m=1 / 3$, the dashed has $m=2 / 3$, and the dotted has $m=1$. The thin solid line is a piecewise linear model that assumes all winds originate with a similar fraction of turbulent kinetic energy to bulk flow kinetic energy, and indicates X-ray efficiency scaling with $\dot{M}$ for adiabatically cooled B-star winds, and in the central domain the efficiency required for $L_{x}$ proportional to bolometric luminosity in O stars, and then $L_{x}$ independent of $\dot{M}$ for H-depleted WR winds, the latter of which provides a reasonable fit to Skinner et al. (2012). The figure shows that $m \sim 2 / 3$ gives a rough reproduction of the overall scaling of efficiency with mass-loss rate if all winds contain a similar fraction of turbulent kinetic energy. 


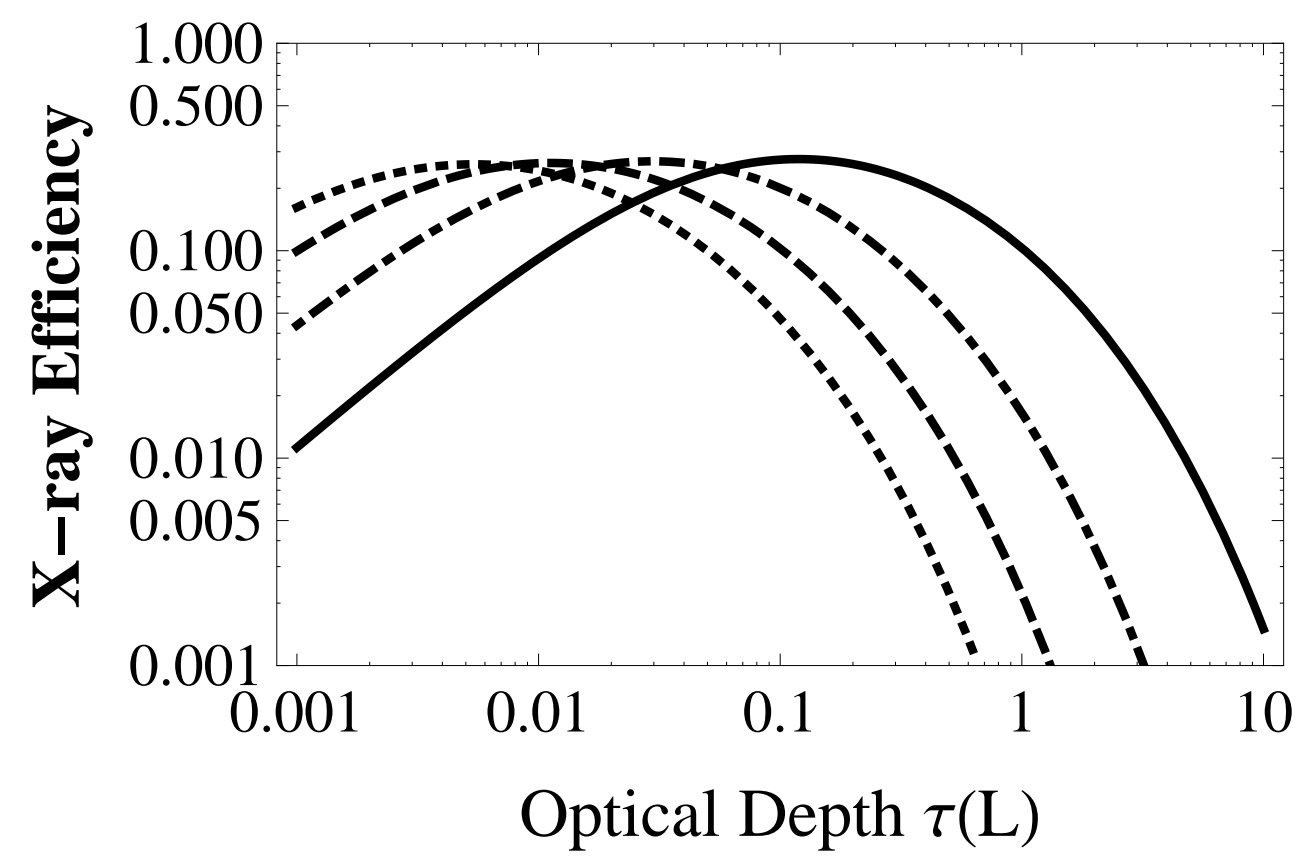

Figure 3: Here the X-ray efficiency $L_{x} / E_{x}$ is plotted as a function of mass-loss rate characterized by $\tau(L)$, for fixed $v_{\infty}$ and assuming $m=2 / 3$, using $\Delta v=v_{\infty} / 2$ (solid curve), $v_{\infty} / 3$ (dot-dashed), $v_{\infty} / 4$ (dashed), and $v_{\infty} / 5$ (dotted). We see that stronger shocks produce more visible X-rays from winds that are optically thick to reabsorption (since hotter emissions emerge better through the reabsorption), whereas winds that are optically thin to reabsorption produce a higher X-ray generation efficiency in weaker shocks (since fast radiative cooling times compete more effectively against adiabatic cooling). 
efficiency $L_{x} / E_{x}$, and we see that emission from large radii is indeed possible in WR winds, but only if $L$ is taken to be at least an order of magnitude larger than the stellar radius. This is not unreasonable, however, because evidence exists (Lepine \& Moffat 1999; Dessart \& Owocki 2005b) that the acceleration zone is similarly stretched by the optically thick character of WR winds. How this occurs, and how it relates to the energization of the turbulence, remains unclear, though progress has been made in simulations that can show very extended acceleration (e.g., Gräfener \& Hamann 2005).

Another important ramification of Fig. 4 is that, in this model, the X-rays from a given shock strength emerge preferentially from a fairly well defined domain, perched near the $\tau=1$ point at the X-ray energy characteristic of that shock strength. Emission at smaller radii is largely reabsorbed, while emission at larger radii is likely squelched by an inability to advect turbulent energy at that shock strength any farther out, given that it is constantly encountering clumps in the terminal wind. This latter effect is more pronounced at higher shocks strengths, such that harder emissions are highly localized in dense winds. This may allow different X-ray lines to provide spatially resolved information about the hot gas in dense winds, information that is of a complementary nature to the tendency for all the emission to arise from the vicinity of the acceleration zone in optically thin winds.

\subsection{Unifying the X-ray emission of $O B$ and WR winds}

The above approach produces a framework for understanding the fraction of turbulent energy in the wind that can be converted to observed X-ray emission. As such, it makes no attempt to establish how much turbulent energy is deposited in the wind by the engine that is ultimately responsible for the X-rays. The intention is to be able to use X-ray observations, in concert with this escape efficiency analysis, to constrain that engine's conversion fraction of bulk kinetic energy flux to thermalized X-ray hot gas. Inferring that conversion fraction could favor or rule out various possible energization mechanisms.

Since the approach here is unification, the initial hypothesis to test is that the engine could exhibit a similar conversion fraction in all these winds, though at present there is no reason to claim this is anything more than a working assumption. Since a nominal expectation is for the X-ray luminosity to be roughly $10^{-7}$ of the stellar luminosity (e.g., Naze et al. 2011), and the kinetic energy flux in $\mathrm{O}$ winds is generally in the range $10^{-3}$ to $10^{-2}$ of the stellar luminosity, a canonical total efficiency for converting wind kinetic 


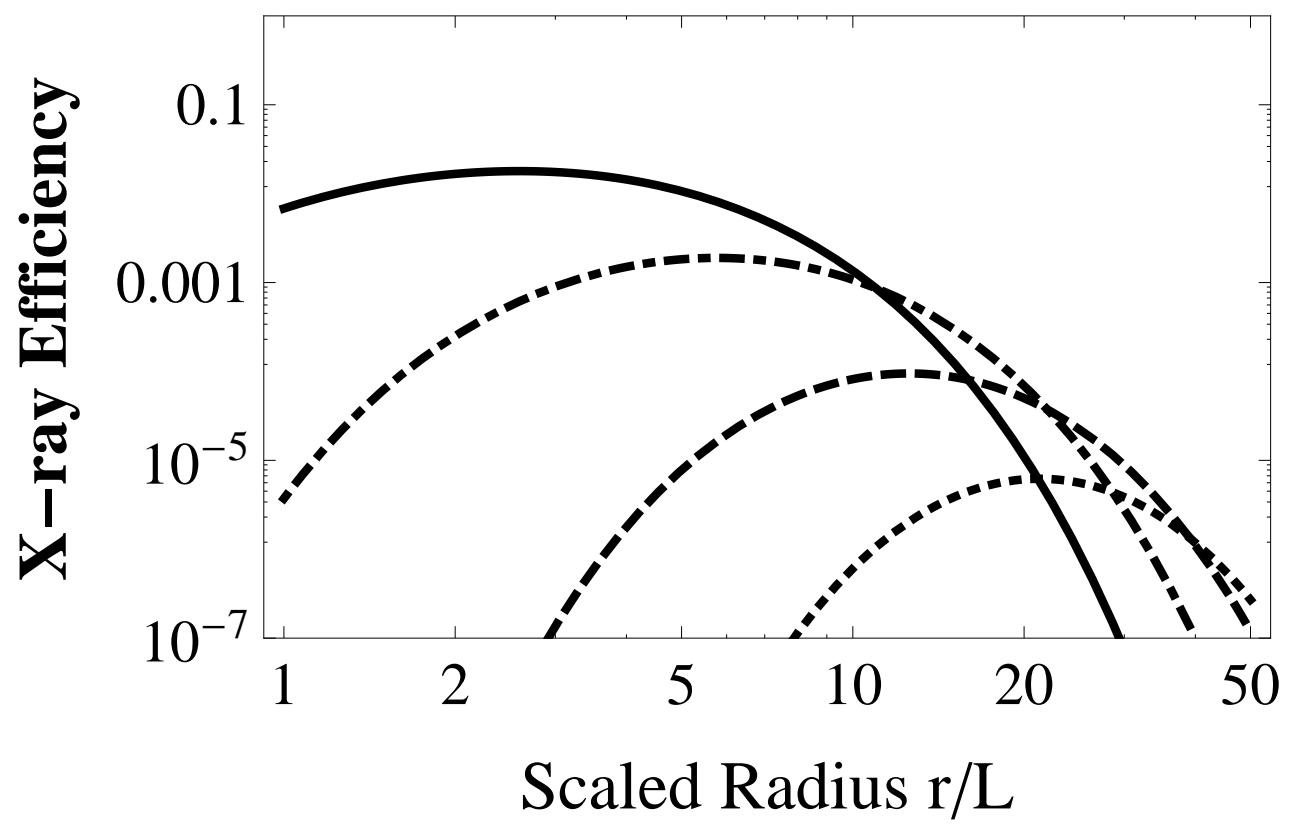

Figure 4: The radial profile of the contribution to $L_{x} / E_{x}$ is plotted in units of scaled radius $z$, at fixed $v_{\infty}=2000 \mathrm{~km} \mathrm{~s}^{-1}$ and a given WR mass-loss rate corresponding to $\tau(L)=10$ at $1 \mathrm{keV}$, for different shock strengths $\Delta v=1000 \mathrm{~km} \mathrm{~s}^{-1}$ (solid), $700 \mathrm{~km} \mathrm{~s}^{-1}$ (dot-dashed), $500 \mathrm{~km} \mathrm{~s}^{-1}$ (dashed), and $400 \mathrm{~km} \mathrm{~s}^{-1}$ (dotted). We see that the hotter emissions from stronger shocks can emerge from deeper in the wind, which is fortunate because their more rapid overtaking speed does not given them time to be advected far out. Weaker shocks can advect farther out, but their cooler emissions are more heavily reabsorbed, and should not contribute strongly to X-rays from the densest winds. Also, the X-ray emergence can only come from large $r \gg R_{*}$ if $L$ is large in WR winds. 
energy to emergent X-rays could be expected to fall in the range $10^{-5}-10^{-4}$ for O stars. In Fig. 2 we see a broad peak in the 10\%-30\% range for the fraction of all the energy that is thermalized into X-ray hot gas that ultimately escapes as X-rays, for $\mathrm{O}$ stars. Hence, for $\mathrm{O}$ stars, we expect that the conversion fraction of bulk kinetic energy into X-ray hot gas is of magnitude $10^{-4}$. In WR winds, one would expect a slower line-driven instability growth rate in the linear regime (Gayley et al. 2005), but the conversion fraction should depend more on the saturation of the turbulence, than on its growth rate, so it is possible that X-ray generation efficiencies could be in that same range for WR winds. However, due to increased reabsorption, the emergent X-ray efficiencies should be less.

\subsection{Application to WR6}

When applied to EZ CMa (WR6), we use an observed X-ray luminosity of $8 \times 10^{32} \mathrm{erg} \mathrm{s}^{-1}$, an inferred wind kinetic energy flux of $6 \times 10^{37} \mathrm{erg} \mathrm{s}^{-1}$, and a bolometric luminosity of $1.6 \times 10^{39} \mathrm{erg} \mathrm{s}^{-1}$ (all from Huenemoerder et al. 2015), to find an $L_{x} / L_{b o l}$ ratio of $5 \times 10^{-7}$ and an efficiency for converting wind kinetic energy into emergent X-rays of $1.7 \times 10^{-5}$. Rough nominal expectations for the model parameters, given the above definitions of $z_{s}$ and $z_{\tau}$, are in this case $z_{s} \sim 3$ (since the characteristic excess speed of the gas entering the shock is about $700 \mathrm{~km} \mathrm{~s}^{-1}$ to heat He-rich gas to temperatures characteristic of $1 \mathrm{keV}$ X-ray production, and the terminal speed is $\sim 2000$ $\mathrm{km} \mathrm{s}^{-1}$ ), and $z_{\tau} \sim 8$ ( is roughly 100, Oskinova et al. 2012). Thus we can see from eq. (11) that the model efficiency for the conversion of sufficiently hypersonic turbulent energy to observable X-ray emission (i.e., accounting for adiabatic cooling, mixing, and reabsorption) is about $5 \%$ if $m=1 / 3$ and $2 \%$ if $m=2 / 3$.

Combining this with a total efficiency of $1.7 \times 10^{-5}$ for converting kinetic energy into observable X-rays, we infer that the conversion fraction for wind kinetic energy into turbulent energy that is sufficiently hypersonic to produce $\mathrm{X}$-ray hot gas is a few times $10^{-4}$. Alternatively, we can assume this conversion fraction is approximately universal for $\mathrm{O}$ stars and WR winds, and estimate the X-ray luminosity of EZ CMa by taking its wind kinetic energy flux of $6 \times 10^{37} \mathrm{erg} \mathrm{s}^{-1}$, multiply by a putative O-star conversion fraction of $3 \times 10^{-4}$ from above to estimate a total energization rate of some $2 \times 10^{34} \mathrm{erg}$ $\mathrm{s}^{-1}$ going into X-ray hot gas. Then eq. (11) gives for $z_{s} \sim 3$ and $z_{\tau} \sim 8$ that for $m$ in the range $1 / 3$ to $2 / 3$, the X-ray luminosity for EZ CMa could be estimated to be in the range 4 to $10 \times 10^{32} \mathrm{erg} \mathrm{s}^{-1}$. That the WR conversion 
fractions are broadly consistent with the conversion fractions deduced for $\mathrm{O}$ stars above, or equivalently, that the estimated EZ CMa X-ray luminosity is broadly consistent with observations, lends credence to the possibility that a similar X-ray energization process could be at work in WR winds as in O-star winds. Determining whether this process is the line-driven instability, or some other mechanism involving a windspeed that varies in space or time, will require more detailed simulations of turbulence in the acceleration of the densest winds.

\section{Conclusions}

Here a simple model is considered for X-ray heating involving a homologous heuristic model that can be scaled over a wide range of wind conditions, with the intention of placing Wolf-Rayet winds into a broader context that includes $\mathrm{OB}$ winds. The purpose is to explore whether or not it is possible that WR winds could be X-ray sources via similar mechanisms as single nonmagnetic OB stars. The key parameter introduced into the model is the correlation length $L$, the length scale of the separation between fast windstreams from the prevailing clumpy terminal wind. This parameter is somewhat arbitrary, yet may be expected to be of order the acceleration zone, since that is the length scale over which the wind acquires its bulk kinetic energy flux, so may also be the length scale involved in turbulent energization. Shocks are assumed to begin forming over this same length scale, which is deeply embedded in the dense winds of $\mathrm{H}$-poor WR winds, so a significant fraction of the X-rays do not escape reabsorption, pushing down the emergent X-ray efficiency. Also, mixing of hot and cool gas is assumed to further reduce the efficiency, extending into the WR domain the need for mixing inferred for $\mathrm{O}$ stars in Owocki et al. (2013).

However, these effects do not quell the X-ray luminosity, because the higher density in WR winds also allows X-rays to be efficiently generated at much larger radii than in $\mathrm{OB}$ winds. Another important effect that contributes to X-ray emission in this model is the observed tendency for the acceleration region in WR winds to be extended, which may draw out the correlation length $L$ sufficiently to forestall the shocking of the fast gas long enough for it to be advected beyond the X-ray reabsorption photosphere.

Thus, if this model is a reasonable description of the actual turbulence in WR winds, then dense winds allow observation of the "tail" of the same $\mathrm{X}$-ray generation mechanism present in $\mathrm{OB}$ winds, merely extended to large 
radius, perhaps even a hundred stellar radii as inferred for WR6 (Oskinova et al. 2012). This spatial tail in the heating could possibly be present in all single nonmagnetic stars, as per the self-similar model assumed here, but is merely undetectable from lower mass-loss rates because of competition from deeper higher density emission that is not reabsorbed in optically thin winds.

The idealization here is essentially a two-component model, where fast gas rams the prevailing wind, but this is merely intended as a simplified version of the kind of clump-clump collisions whose importance was stressed originally by Feldmeier et al. (1997), where here we have essentially fast and slow "clumps" that comprise the bulk of the wind. The model is stochastic but one dimensional, yet would function similarly in higher dimensions if the wind and all its embedded turbulence simply advects radially, and the clump-clump collisions are also radial. Rotation of the star breaks the spherical symmetry, leading potentially to structures known as co-rotating interaction regions (Mullan 1984; Morel, St-Louis, \& Marchenko 1997; Ignace et al. 2013), which could steepen into shocks at large radii. Since the CIR-type density features seen in UV absorption stem from more gradual velocity variations such as kinks rather than discontinuities (Cranmer \& Owocki 1996), the connection between the CIRs that appear in the UV, and the shocks that produce the X-rays, is not direct. Still, if denser winds allow observation of X-ray heating at larger radii, the results here suggest that CIR features might show up in X-rays more in WR winds than they would in OB winds.

A key feature of the unified picture explored here is that the thick and thin winds tell a complementary story, and both are required to spatially resolve the extended heating responsible for X-ray generation in single nonmagnetic hot stars. Hence, one general prediction of this unified model is that the best way to observe the features of any given wind is to observe X-ray lines at energies that are barely optically thick at that radius. Another expected feature due to the energy dependence of reabsorption opacity is that harder emissions would be relatively more prevalent in dense winds, owing to the fact that they can emerge from a wider domain than softer emissions.

The general scheme presented here does not produce any obvious contradictions with data, so long as $L$ can be long in WR winds. Indeed, without mixing of hot and cool gas, X-ray efficiency in WR winds seem too high, even in winds with substantial optical depth to reabsorption of $1 \mathrm{keV}$ X-rays of order 10 at $r=L$, which might produce total optical depth over the whole wind greater than 100. Hence WR winds may require mixing for the same 
reason that O-type winds appear to, to keep $L_{x}$ from rising too rapidly as $\dot{M}$ rises (Owocki et al. 2013).

The purpose of the heuristic results here are to provide a kind of quantitative benchmark against which to compare the results of more complete simulations. The overall scheme may help us understand why Skinner et al. (2012) find that low- $v_{\infty}$ terminal speed WR winds have low X-ray efficiency, on the grounds that such winds cannot advect the turbulent gas outside the reabsorption photosphere. It also may help explain the tendency found in Skinner et al. (2012) for all H-poor WR winds to have similar X-ray luminosities, up to fairly high mass-loss rates, because in this picture, stronger reabsorption compensates for higher kinetic energy flux in winds with high mass flux.

The author would like to thank R. Ignace for many helpful conversations on this topic, and D. Huenemoerder, L. Oskinova, and W.-R. Hamann for invaluable data and collaboration. The two referees also submitted very useful comments and suggestions, but one of them, S. P. Owocki, made suggestions that so completely altered the final result as to rise to the level of contribution of a coauthor. Almost every conclusion of merit in the new version is in direct response to that comprehensive critique. This work was supported in part by NASA grants NNX11AF83G to the University of Iowa and GO3-14003C via the Smithsonian Astrophysical Observatory.

\section{References}

Antokhin, I. I., Owocki, S. P., \& Brown, J. C., A steady, radiative-shock method for computing X-ray emission from colliding stellar winds in close, massive-star binaries, ApJ, 611, 434-451, 2004.

Cranmer, S. R. \& Owocki, S. P., Hydrodynamical simulations of corotating interaction fegions and discrete absorption components in rotating O-star winds, ApJ, 462, 469-488, 1996.

Dessart, L. \& Owocki, S. P., 2D simulations of the line-driven instability in hot-star winds II. Approximations for the 2D radiation force, A\&A, 437, 657666, 2005a.

Dessart, L. \& Owocki, S. P., Inferring hot-star-wind acceleration from Line Profile Variability, A\&A, 432, 281-294, 2005 b. 
Feldmeier, A., Puls, J., \& Pauldrach, A. W. A., A possible origin for X-rays from O stars, A\&A, 322, 878-895, 1997.

Gayley, K. G. \& Owocki, S. P., Line-driven instability growth rates in WolfRayet winds, ApJ, 446, 801-811, 1995.

Gräfener, G. \& Hamann, W.-R., Hydrodynamic model atmospheres for WR stars. Self-consistent modeling of a WC star wind, A\&A, 432, 633-645, 2005.

Hamann, W.-R., Gräfener, G., \& Liermann, A., The galactic WN stars. Spectral analyses with line-blanketed model atmospheres versus stellar evolution models with and without rotation, A\&A, 457, 1015-1031, 2006.

Huenemoerder, D. P., Gayley, K. G., Hamann, W.-R., Ignace, R., Nichols, J. S., Oskinova, L., Pollock, A. M. T., Schulz, N. S., \& Shenar, T., Probing Wolf-Rayet winds: Chandra/HETG X-ray spectra of WR6, ApJ, in press, 2015.

Ignace, R., Gayley, K. G., Hamann, W.-R., Huenemoerder, D. P., Oskinova, L. M., Pollock, A. M. T., \& McFall, M., The XMM-Newton/Epic X-ray light curve analysis of WR6, ApJ, 775, 29-40, 2013.

Kee, N. D., Owocki, S. P., \& ud-Doula, A., Suppression of X-rays from radiative shocks by their thin-shell instability, MNRAS, 438, 3557-3567, 2014.

Krticka, J., Feldmeier, A., Oskinova, L. M., Kubat, J., Hamann, W.-R., Xray emission from hydrodynamical simulations of non-LTE wind models, A\&A, 508, 841-848, 2009.

Lepine, S. \& Moffat, A. F. J., Wind Inhomogeneities in Wolf-Rayet Stars. II. Investigation of Emission-Line Profile Variations, ApJ, 514, 909-931, 1999.

Leutenegger, M. A., Cohen, D. H., Zsargo, J., Martell, E. M., MacArthur, J. P., Owocki, S. P., Gagne, M., \& Hillier, D. J., Modeling broadband X-ray absorption of massive star winds, ApJ, 719, 1767-1774, 2010.

MacFarlane, J. J., Cassinelli, J. P., Welsh, B. Y., Vedder, P. W., Vallerga, J. V., Waldron, W. L., Predicted extreme-ultraviolet line emission for nearby main-sequence B stars, ApJ, 380, 564-574, 1991. 
Morel, T., St-Louis, N., \& Marchenko, S. V., Optical spectoscopy of EZ Canis Majoris: Indication for large-scale structures in a Wolf-Rayet wind, ApJ, 482, 470-489, 1997.

Mullan, D., Corotating Interaction Regions in Stellar Winds, ApJ, 283, 303$312,1984$.

Naze Y., Broos P. S., Oskinova L., Townsley L. K., Cohen D., Corcoran M. F., Evans N. R., Gagne M., Moffat A. F. J., Pittard J. M., Rauw G., udDoula A., Walborn N. R., Global X-ray properties of the O and B stars in Carina, ApJS, 194, 7-26, 2011.

Oskinova, L. M., Gayley, K. G., Hamann, W.-R., Huenemoerder, D. P., Ignace, R., \& Pollock, A. M. T., High-resolution X-ray spectroscopy reveals the special nature of Wolf-Rayet star winds, ApJ, 747, L25-L30, 2012.

Owocki, S. P., Sundqvist, J. O., Cohen, D. H., \& Gayley, K. G., Thin-shell mixing in radiative wind-shocks and the Lx Lbol scaling of O-star X-rays, MNRAS, 429, 3379-3389, 2013.

Runacres, M. C. \& Owocki, S. P., The outer evolution of instability-generated structure in radiatively driven stellar winds, A\&A, 381, 1015-1025, 2002.

Skinner, S. L., Zhekov, S. A., Gudel, M., Schmutz, W., \& Sokal, K., New X-Ray Detections of WNL Stars, AJ, 143, 116-123, 2012.

Sundqvist, J. O., Owocki, S. P., Cohen, D. H., Leutenegger, M. A., \& Townsend, R. H. D., A generalized porosity formalism for isotropic and anisotropic effective opacity and its effects on X-ray line attenuation in clumped O star winds, MNRAS, 420, 1553, 2012. 